University of Nebraska - Lincoln

DigitalCommons@University of Nebraska - Lincoln

May 1995

\title{
Nanoscale Visualization and Control of Ferroelectric Domains by Atomic Force Microscopy
}

Oleg Kolosov

Joint Research Center for Atom Technology, Angstrom Technology Partnership and National Institute for Advanced Interdisciplinary Research, 1-1-2, Higashi, Tsukuba, Ibaraki 305, Japan,

o.kolosov@lancaster.ac.uk

Alexei Gruverman

University of Nebraska-Lincoln, agruverman2@unl.edu

Jun Hatano

Science University of Tokyo

Koichiro Takahashi

National Institute for Research in Inorganic Materials, I - I Namiki, Tsukuba, Ibaraki 305, Japan

Hiroshi Tokumoto

Joint Research Center for Atom Technology, Angstrom Technology Partnership and National Institute for Advanced Interdisciplinary Research, 1-1-2, Higashi, Tsukuba, Ibaraki 305, Japan

Follow this and additional works at: https://digitalcommons.unl.edu/physicsgruverman

Part of the Physics Commons

Kolosov, Oleg; Gruverman, Alexei; Hatano, Jun; Takahashi, Koichiro; and Tokumoto, Hiroshi, "Nanoscale Visualization and Control of Ferroelectric Domains by Atomic Force Microscopy" (1995). Alexei Gruverman Publications. 39.

https://digitalcommons.unl.edu/physicsgruverman/39

This Article is brought to you for free and open access by the Research Papers in Physics and Astronomy at DigitalCommons@University of Nebraska - Lincoln. It has been accepted for inclusion in Alexei Gruverman Publications by an authorized administrator of DigitalCommons@University of Nebraska - Lincoln. 


\title{
Nanoscale Visualization and Control of Ferroelectric Domains by Atomic Force Microscopy
}

\author{
Oleg Kolosov, ${ }^{1,2, *}$ Alexei Gruverman, ${ }^{3, \dagger}$ Jun Hatano, ${ }^{4}$ Koichiro Takahashi, ${ }^{3}$ and Hiroshi Tokumoto ${ }^{1}$ \\ ${ }^{1}$ Joint Research Center for Atom Technology, Angstrom Technology Partnership and National Institute for Advanced \\ Interdisciplinary Research, 1-1-2, Higashi, Tsukuba, Ibaraki 305, Japan \\ ${ }^{2}$ Mechanical Engineering Laboratory, Namiki 1-2, Tsukuba, Ibaraki 305, Japan \\ ${ }^{3}$ National Institute for Research in Inorganic Materials, 1-1 Namiki, Tsukuba, Ibaraki 305, Japan \\ ${ }^{4}$ Department of Materials Science and Technology, Science University of Tokyo, Noda, Chiba 278, Japan
}

(Received 17 March 1994)

\begin{abstract}
The nanoscale visualization and control of domain structure with atomic force microscopy (AFM) in the ferroelectric crystal guanidinium aluminum sulfate hexahydrate is reported. The origin of the domain contrast in the topographic of AFM images is explained by the piezoelectric deformation of the crystal surface in the internal electric field. The domain structure was modified by applying a voltage to the conductive AFM tip. The dynamics of domain growth has been directly observed for the first time with a resolution of $10 \mathrm{~nm}$.
\end{abstract}

PACS numbers: 77.80.Dj, 85.42.+m, 85.50.Ly

One of the main features of ferroelectrics which singles them out among other materials is the possibility of changing the direction of spontaneous polarization under an applied electric field. Over the past few years, interest in ferroelectrics has essentially increased as a result of recent achievements in the processing of ferroelectric thin films and their wide application in various electronic and optoelectronic devices. Recently the possibility of imaging ferroelectric domains with atomic force microscopy (AFM) [1,2] in the micron range has been reported $[3,4]$. AFM could be a powerful tool for the nanoscale study of domain structure, but a lack of understanding of the origin of ferroelectric domain contrast in AFM obscures the image interpretation. An attractive prospect for AFM, but not yet realized, would be direct nanoscale imaging of domains during their switching. This would allow one to directly study the nature of ferroelectric behavior.

In the present paper an analysis of the basics of ferroelectric domain contrast during contact (repelling force) AFM imaging is given. Using a conductive cantilever under variable electric potential, a controllable local switching of polarization in the ferroelectric crystal was realized and the dynamics of domain nucleation, growth, and interaction was studied with nanoscale resolution.

To investigate the domain contrast in AFM we used a guanidinium aluminum sulfate hexahydrate (GASH) crystal, chemical formula $\mathrm{C}\left(\mathrm{NH}_{2}\right)_{3} \mathrm{Al}\left(\mathrm{SO}_{4}\right)_{2} \cdot 6 \mathrm{H}_{2} \mathrm{O}$. GASH is a uniaxial ferroelectric, belonging to the trigonal crystal system (space group $P 31 \mathrm{~m}$ ) [5]. The spontaneous polarization $P_{s}$ is about $0.35 \mu \mathrm{C} / \mathrm{cm}^{2}$, the dielectric constant $\varepsilon_{c}$ along the polar axis is 15 , and the coercive field is about $1.5 \times 10^{5} \mathrm{~V} / \mathrm{m}$ [5]. GASH has a perfect cleavage plane normal to the polar axis $c$, which is quite suitable for studying the domain structure. It has been studied using various techniques, among them scanning electron microscopy [6], powder deposition [7], pyroelectric probe imaging [8], nematic liquid crystals [9], and, recently, by AFM with micrometer resolution $[3,4]$.

The crystal plates, which were cleaved in air, were about $0.3 \mathrm{~mm}$ thick. They were investigated in an ambient environment using a Nanoscope III scanning probe microscope operating in AFM topography and friction modes. To distinguish the contrast associated with topography from that related to electrostatic tip charging, we used two types of cantilevers - a standard one with a nonconductive $\mathrm{Si}_{3} \mathrm{~N}_{4}$ tip and a gold-coated conductive tip and cantilever. Both cantilevers had a rigidity of $0.09 \mathrm{~N} / \mathrm{m}$ and tips with a curvature radius $R$ of about $100 \mathrm{~nm}$. During imaging with the conductive tip we applied a dc potential $V$ of up to $\pm 5 \mathrm{~V}$ to the bottom electrode, whereas the tip was held to zero potential.

The AFM images of the GASH surface showed monatomic cleavage steps of about $0.8 \mathrm{~nm}$ in height and of $1-10 \mu \mathrm{m}$ in separation. The well known GASH domain structure in the form of rows of cylindrical domains [10] together with a configuration consisting of domains of one sign embedded into the larger domain of opposite sign [Fig. 1(a)] was observed in the topography image. The domains appeared as circular protrusions or depressions with a $100-500 \mathrm{~nm}$ diameter and a 10-50 nm height. There was an attempt to associate the domain contrast in AFM with the electrostatic interaction between polarization and tip charges [4]. Such an explanation is reasonable for the noncontact mode [3] where the conductive tip, biased by electric potential, is easily deflected by the stray electric field of the domain. Nevertheless, in a contact mode, a tip-surface distance is almost fixed due to the strong short-range tip-surface repulsive interaction, resulting in imaging of mainly the surface topography. Moreover, there was no essential difference in the topography contrast observed for the nonconductive cantilever and the electrically grounded 

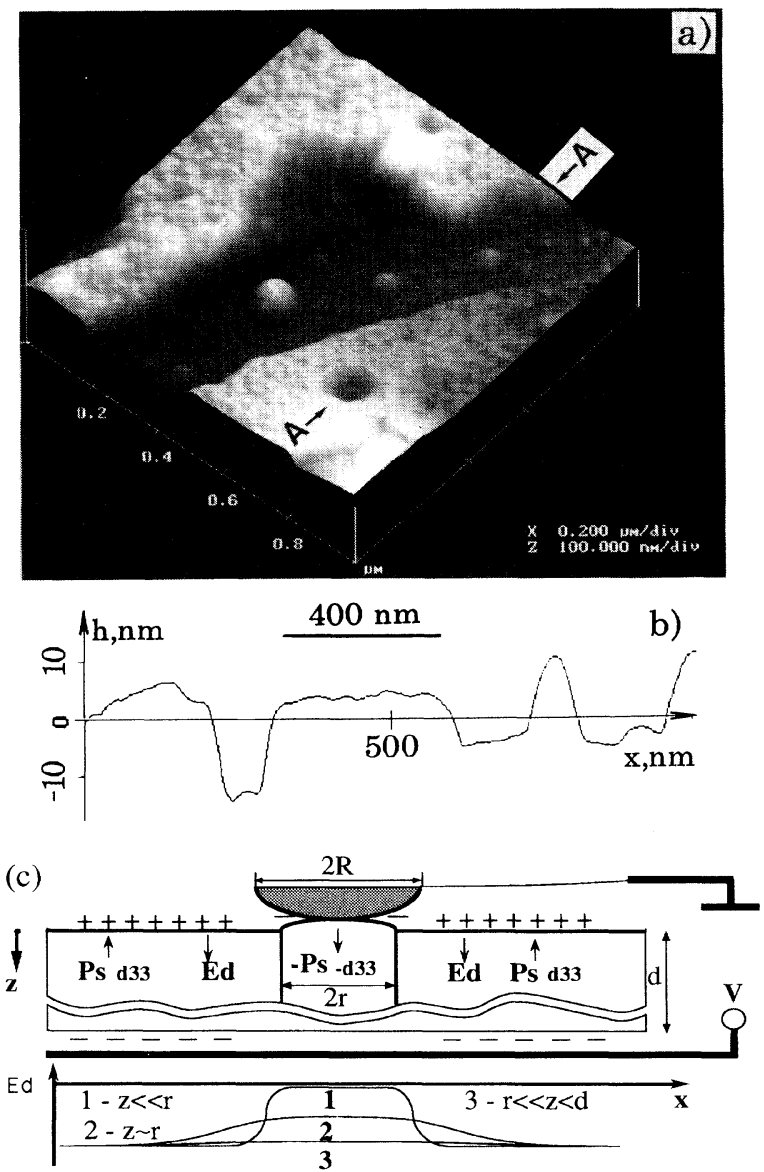

FIG. 1. (a) Domain structure in GASH revealed by AFM, operating in topography mode. (b) Topography profile on the cleavage surface of GASH crystal due to the piezoelectric deformation in domains of opposite signs. (c) Schematic diagram, illustrating the mechanism of domain contrast, and the distribution of depolarization field $E_{d}$ in the vicinity of through domain.

conductive one. The explanation of the contrast due to tip charging has consequently been ruled out.

The sign of topography variation (protrusion or depression) in the area much smaller than the sample thickness depended on the domain sign [Fig. 1(a) and 1(b)], suggesting the linear response of the topography on the domain orientation. The possible linear mechanism, connecting mechanical deformation and electrical parameters which vary with the domain orientation, is piezoelectricity. There are two parameters, involved in the piezoelectric interaction, namely, the internal electric field $E_{d}$ and the piezoelectric constant. From electrostatic considerations it is obvious that in the area of elongated domain of radius $r$, much smaller than crystal thickness $d$ the depolarization field $E_{d}$ decreases only in the adjacent vicinity below the crystal surface. This field has a coinciding sign within and outside the domain [Fig. 1(c)]. However, the piezoelectric constant $d_{33}$ changes its sign with the reversal of the domain orientation [11]. Therefore, it is $d_{33}$ that causes the experimentally revealed topography variations which are sensitive to the domain sign. The profile variation on $h$ can be approximately estimated as $h \approx 2 d E_{d} d_{33}$. Since on average $E_{d}=2.5 \times 10^{7} \mathrm{~V} / \mathrm{m}$ and $d_{33}=2 \times 10^{-12} \mathrm{~m} / \mathrm{V}$ [11], we obtain a value for $h$ of about $30 \mathrm{~nm}$, and this is of the same order as the experimentally measured values. Therefore, the contrast of ferroelectric domains in the topography AFM mode is consistently explained by the piezoelectric stretching and compressing of domains of opposite sign in the internal electrical field of the ferroelectric crystal.

To further verify that surface electrostatic charges do not account for the observed topography contrast, we applied a dc voltage between the conductive tip and bottom electrode. As we expected, there was no effect on the topography contrast, thereby proving the piezoelectric origin of the surface topography. At the same time, this type of experiment led us to the observation of an interesting phenomenon.

We have found that an applied voltage of a particular sign opposite to the initial ferroelectric polarization (domain orientation) of the crystal created new domains, whereas the potential of the opposite sign erased these or already existing domains. In Fig. 2(a) the rows of domains of regular round shape (cf. [10]) were presented on the freshly cleaved surface with clear monatomic steps. In Fig. 2(b), an array of new domains of radius $r$ as small as $200 \mathrm{~nm}$, written by an AFM tip, are shown. Stable domains were created by the application of voltage pulses of $3 \mathrm{~V}$ amplitude and $4 \mathrm{sec}$ duration while the tip was positioned at $500 \mathrm{~nm}$ spaced sites. A genuine stability and reproducibility of images of new domains during AFM imaging, their noticeably regular round shape [very similar to the already existing domains shown in Fig. 2(a)], and the absence of image "ghosts" ruled out possible interpretation of such features as "contaminants" as well as modification of tip geometry.

Because of inherent hysteresis of the piezoelectric scanner of the AFM it was impossible to point the tip

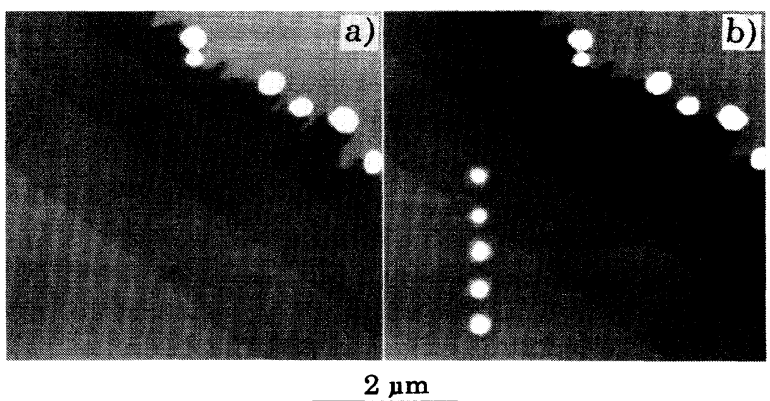

FIG. 2. AFM topography images before (a) and after (b) the application of local electric field by the AFM tip, resulting in the creation of an array of five circular domains. Diagonal grey bands are the monatomic lattice steps of about $0.8 \mathrm{~nm}$ height. 
exactly on the position of a created domain to erase it. To overcome this, domain erasure was demonstrated by the application of the opposite potential to the tip while it was scanned over the area of existing domains.

To quantitatively describe the domain dynamics in an applied field, we considered the elementary process of domain nucleation and growth. The electric field, produced by the tip of radius $R$, positioned close to the surface of the object with dielectric permittivity $\varepsilon_{a} \gg 1$, can be estimated by its charge $q \approx 4 \pi \varepsilon_{a} \varepsilon_{0} R V$, acquired due to the applied voltage $V$ [Fig. 1(c)], as

$$
E \approx q /\left[4 \pi \varepsilon_{a} \varepsilon_{0}(z+R)^{2}\right] \approx R V /(z+R)^{2},
$$

where $z$ is the distance from the surface. Therefore, the electric field, produced by the tip near the surface $E_{s}(z \ll R)$, in the present case, can be estimated as $E_{s} \approx$ $V / R \approx 3 \times 10^{7} \mathrm{~V} / \mathrm{m}$. This field is much higher than the coercive field $E_{c}$, so one could expect the formation of a domain nucleus of reversed polarization on the sample surface faced to the tip. We assume that this nucleus is a half of a prolate spheroid of length $l$ and of radius $r_{n}$, aligned in the polar direction. Because of the growth of the reversed domain the energy of the crystal will be changed by an amount [12]

$$
U=-\frac{4}{3} E P_{s} \pi r_{n}^{2} l+\frac{1}{2} \pi^{2} r_{n} l \sigma+U_{d},
$$

where the expression for the depolarization energy $U_{d}$ was given by Landauer [13], and has the form

$$
U_{d}=\frac{16 P_{s}^{2} r_{n}^{2} l^{\prime}\left(n^{2}-1\right)\left\{\frac{1}{2} n \log [(n+1 / n-1)]-1\right\}}{3\left(e_{a} e_{c}\right)^{1 / 2}},
$$

where $n=l^{\prime} /\left(l^{2}-r_{n}^{2}\right)$, and $l^{\prime}=l\left(e_{a} e_{c}\right)^{1 / 2}$.

The first term in Eq. (3) is associated with the interaction of the applied field with the ions and the second one is the energy required to create a new domain wall. For numerical estimations we used $\sigma=0.3 \times 10^{-3} \mathrm{~J} / \mathrm{m}^{2}$ [14]. For $E_{s}=3 \times 10^{7} \mathrm{~V} / \mathrm{m}$ we find that $U$ has a maximum at $r_{n} \approx 15 \mathrm{~nm}$, with a slight dependence on the domain length $l$, thereby giving the radius of the critical nucleus. When formed the nucleus grows through the sample in the polar direction. This subsequent growth proceeds in a field decreasing as $1 / z$ with depth [Eq. (1)]. It is easy to evaluate, using Eq. (2), that for a domain with radius $200 \mathrm{~nm}$ the field strength required to move the domain forward in the polar direction has to exceed $900 \mathrm{~V} / \mathrm{cm}$. This critical field is achieved at a depth $z$ of about $50 \mu \mathrm{m}$, which corresponds to the vertical size of the grown domain. The estimations of nucleus size as well as of electric field, required for domain growth, are consistent with our experimental results and show that the control of the domains on the nanometer scale can be easily provided by AFM.

To investigate the dynamics of the domain growth we acquired AFM images while the scanning tip was under potential, generating domain growth. The appearance of unusual triangular domains in GASH (Fig. 3) has been reported for the first time, whereas circular or shapeless domains are commonly observed at room temperature $[6,10]$. The walls of triangular domains were perpendicular to the crystallographical $a$ axes of the crystal and were rotated by $30^{\circ}$ with respect to the cleavage steps. It was directly observed that sidewise growth of domains proceeded through nucleation on the domain wall [Fig. 3(d)]. The nuclei had a radii of about $20 \mathrm{~nm}$ which agreed fairly well with our estimations. It was also found that the domains that developed earlier encouraged the formation of new ones by the generation of the nuclei in the direction of the crystallographic $a$ axis [Fig. 3(b)], so that new domains appear at some distance from the parent domains [Fig. 3(c)].

Previously, the appearance of triangular domains in lead germanate was explained by a change of the mechanism of sidewise $180^{\circ}$ wall motion in a pulsed field while taking into account the anisotropy of wall energy [15]. Apparently, similar switching conditions had been realized in our experiment, where the scanning tip under the constant potential was equivalent to the application of short pulses of an electric field to a given point on the crystal surface. It was shown [14] that in equilibrium the preferential orientation of the $180^{\circ}$ wall in GASH is perpendicular to the symmetry plane with a substantially lower anisotropy of wall energy. However, in the nonequilibrium switching conditions caused by a pulsed field, this anisotropy might be of much importance, providing the formation of nuclei with minimum surface energy, that is, on the walls in the directions of the $a$ axes.

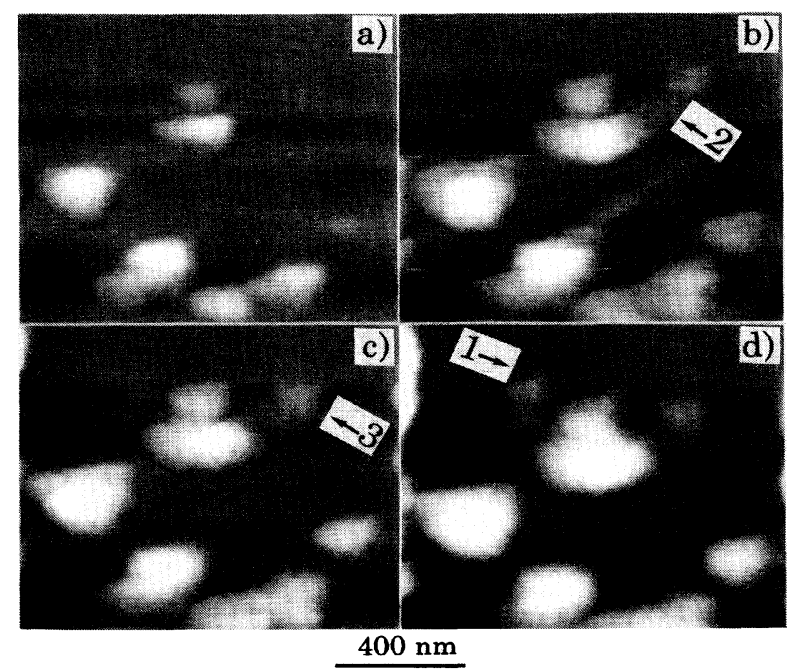

FIG. 3. AFM images, illustrating the dynamics of domain growth during continuous scanning under the voltage applied to the tip. From the series of subsequent images (a)-(d) it is possible to observe the domain nucleation on the walls (1) and "remote generation" (2) of the daughter domain (3) and growth of triangular domains. 
This mechanism can lead to the growth of triangular domains.

In conclusion, the nanoscale visualization and control of the ferroelectric domain structure are reported. We demonstrated the visualization of a domain structure of GASH in AFM topography images with a resolution of about $10 \mathrm{~nm}$. The origin of the domain contrast was discussed and a physical mechanism of contrast was proposed, based on the piezoelectric deformation of the crystal surface. We also directly demonstrated the ability to control the domain structure with AFM by applying an electric potential to the scanning tip. The dynamics of domain growth was directly observed with a nanoscale resolution.

The authors appreciate the invaluable help of Dr. M. Suzuki of NAIR, who kindly provided the excellent SPM equipment for experiments. O.K. also acknowledges the support of Dr. K. Yamanaka of MEL, and expresses gratitude for the creative hands-on introduction to multiprobe scanning microscopy.

*Present address: Department of Materials, University of Oxford, Parks Road 3PH, Oxford, UK;

Electronic address: kolosov@vax.ox.ac.uk On leave of absence from Institute of Chemical Physics, Russian
Academy of Science, Moscow, Russia.

${ }^{\dagger}$ On leave from Institute of Physics and Applied Mathematics, Ural State University, Ekaterinburg, Russia. Electronic address: alexei@ceram.nirim.go.jp

[1] G. Binnig, C. F. Quate, and Ch. Gerber, Phys. Rev. Lett. 56, 930 (1986).

[2] D. Sadrid and V. Elings, J. Vac. Sci. Technol. B 9, 431 (1991).

[3] F. Saurenbach and B. D. Terris, Appl. Phys. Lett. 56, 1703 (1990).

[4] R. Luthi et al., Surf. Sci. 285, L498 (1993).

[5] A. N. Holden et al., Phys. Rev. 98, 546 (1955).

[6] R. Le Bihan et al., Ferroelectrics 55, 189 (1984).

[7] G. L. Pearson and W. L. Feldman, J. Phys. Chem. Solids 9, 28 (1959).

[8] F. Aikawa et al., Ferroelectrics 95, 199 (1989).

[9] L. I. Dontzova, N. A. Tikhomirova, and L. A. Shuvalov, Ferroelectrics 97, 87 (1989).

[10] L. Szczesniak et al., Phys. Status Solidi (a) 88, 93 (1985).

[11] I. S. Zheludev, Physics of Crystalline Dielectrics (Plenum, New York, 1971).

[12] R. C. Miller and G. Weinreich, Phys. Rev. 117, 1460 (1960).

[13] R. Landauer, J. Appl. Phys. 28, 227 (1957).

[14] F. Suda, J. Hatano, and H. Futama, Phys. Status Solidi (a) 95, 505 (1986).

[15] V. Ya. Shur, A. L. Gruverman, and E. L. Rumyantsev, Ferroelectrics 111, 123 (1990). 

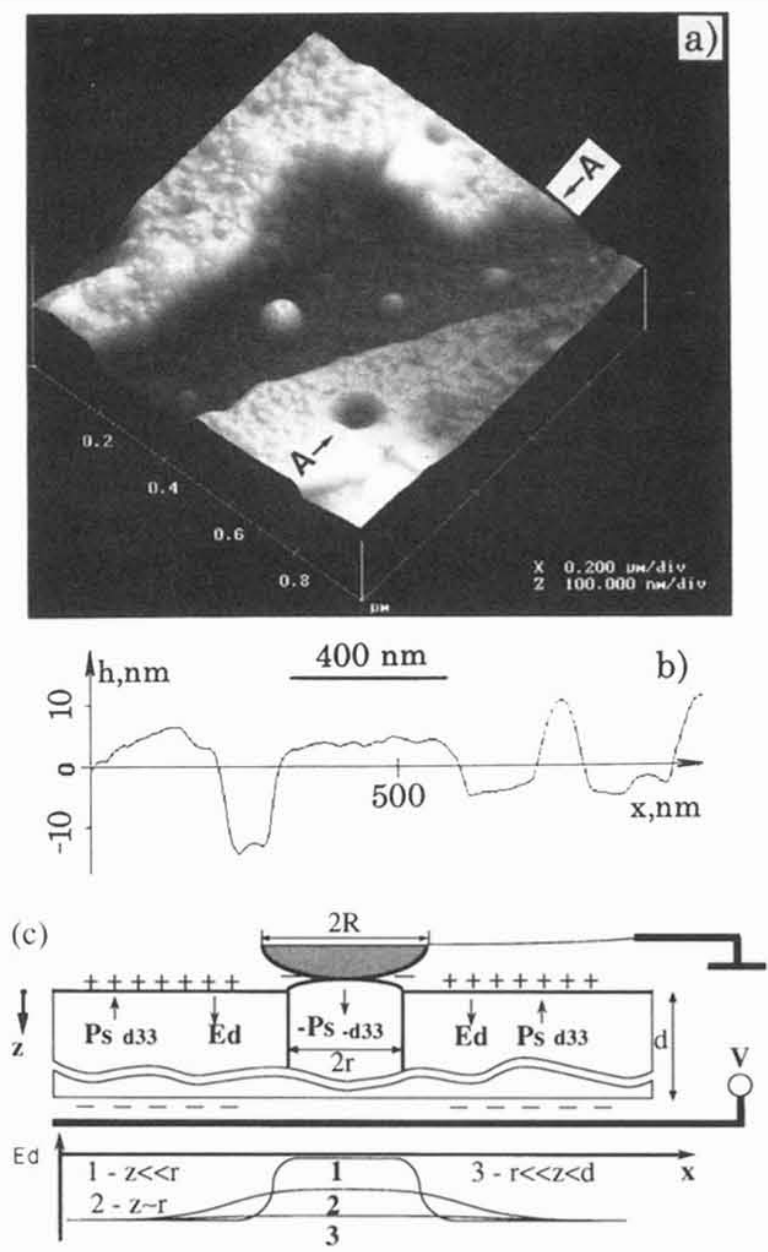

FIG. 1. (a) Domain structure in GASH revealed by AFM, operating in topography mode. (b) Topography profile on the cleavage surface of GASH crystal due to the piezoelectric deformation in domains of opposite signs. (c) Schematic diagram, illustrating the mechanism of domain contrast, and the distribution of depolarization field $E_{d}$ in the vicinity of through domain. 


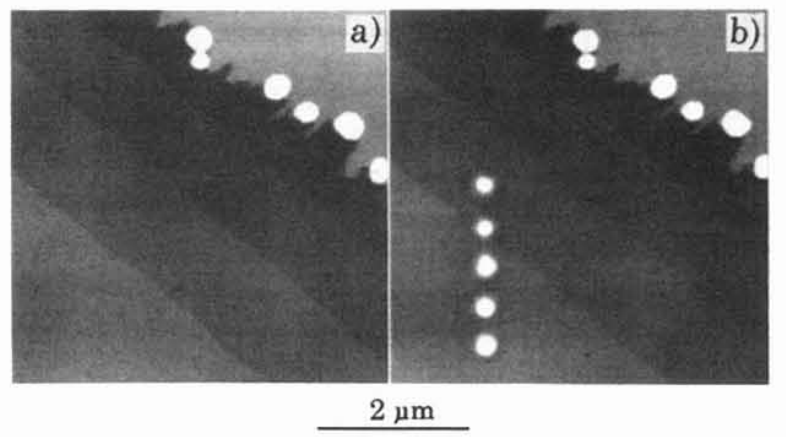

FIG. 2. AFM topography images before (a) and after (b) the application of local electric field by the AFM tip, resulting in the creation of an array of five circular domains. Diagonal grey bands are the monatomic lattice steps of about $0.8 \mathrm{~nm}$ height. 

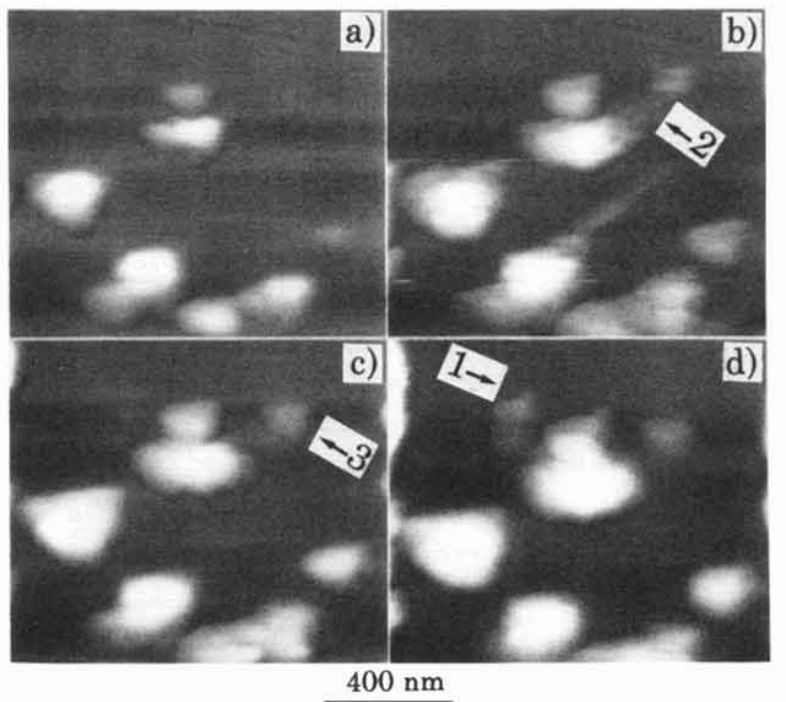

FIG. 3. AFM images, illustrating the dynamics of domain growth during continuous scanning under the voltage applied to the tip. From the series of subsequent images (a)-(d) it is possible to observe the domain nucleation on the walls (1) and "remote generation" (2) of the daughter domain (3) and growth of triangular domains. 